\title{
Hand Hygiene Policy for a Tertiary Care Hospital
}

\author{
${ }^{1}$ Sheetal Singh, ${ }^{2}$ Shakti Kumar Gupta, ${ }^{3}$ Sanjay Arya, ${ }^{4}$ Vijay Aggarwal, ${ }^{5} \mathrm{~T}$ Thuilephy
}

\begin{abstract}
Hand hygiene diminishes the carriage of potential pathogens on the hands. It results in reduction in patient morbidity and mortality from nosocomial infection. Eighty percent of nosocomial disease transmission is thought to be via hands. The purpose of this study is to provide policy with regard to hand hygiene which can be followed in tertiary care hospitals. It was a descriptive cross-sectional study carried out between April and August 2013. The study population included doctors, nursing personnel, paramedical staff and quality managers of tertiary care hospital from public and private hospitals. Checklist was made after an exhaustive review of literature which was then improvised. Validation of the checklist was done by experts in infection control in various private and public hospitals. Subsequently, interaction was done with study population against the back drop of the checklist and hand hygiene policy was formulated.
\end{abstract}

Keywords: Hand hygiene agents, Hand hygiene practices, Hand washing Techniques.

How to cite this article: Singh S, Gupta SK, Arya S, Aggarwal V, Thuilephy T. Hand Hygiene Policy for a Tertiary Care Hospital. Int J Res Foundation Hosp Healthc Adm 2015;3(2):103-109.

\section{Source of support: Nil}

Conflict of interest: None

\section{INTRODUCTION}

Most healthcare-associated infections (HAI) are thought to be transmitted by the hands of healthcare providers (HCPs) through direct contact, mainly when the hands of HCPs transfer microorganisms between individuals or between individuals and the environmental reservoir.

\footnotetext{
${ }^{1}$ Senior Resident, ${ }^{2}$ Medical Superintendent, ${ }^{3}$ Professor

${ }^{4}$ General Manager, ${ }^{5}$ Quality Nurse

${ }^{1,3}$ Department of Hospital Administration, All India Institute of Medical Sciences, New Delhi, India

${ }^{2}$ All India Institute of Medical Sciences, Dr Rajendra Prasad Centre for Ophthalmic Sciences, New Delhi, India

${ }^{4}$ Operations Unit, Max Health Care, New Delhi, India

${ }^{5}$ Grade II Staff, Dr Rajendra Prasad Centre for Ophthalmic Sciences, All India Institute of Medical Sciences, New Delhi, India
}

Corresponding Author: Sheetal Singh, Senior Resident Department of Hospital Administration, All India Institute of Medical Sciences, New Delhi, India, Phone: 01126593308 e-mail: sheetalsingh2003@hotmail.com
It has long been known that hand hygiene among HCPs plays a central role in preventing the transmission of infectious agents. ${ }^{1}$

In developed countries, HAI affects about 5 to $15 \%$ of hospitalized patients. ${ }^{6}$ The rate is higher among those in intensive care units (ICUs) between 9 and 37\%.6,7 The incriminating organisms are often microbial isolates of resistant organisms. ${ }^{8}$ Hospitals in Europe Link for Infection Control through Surveillance (HELICS) estimate millions of extra days of hospital stay and huge economic burden..$^{910}$ In USA, the estimated HAI incidence rate in the year 2004 was $4.5 \%$ with a fatality rate of $5 \%$ and a huge economic impact. ${ }^{11,12}$ Poor hand hygiene among healthcare workers was identified as one of the major causes of the infections. ${ }^{1}$

Hands often act as vectors that carry disease-causing pathogens from person to person, either through direct contact or indirectly via surfaces. Humans can spread bacteria by touching other people's hand, hair, nose, and face, hands that have been in contact with human or animal feces, bodily fluids like nasal excretions, and contaminated foods or water can transport bacteria, viruses and parasites to unwitting hosts.

The value of hand washing for the prevention of cross infection was first observed in the middle of the 19 century. This practice especially when done with soap can remove agents of infection both at the time they were emitted from the primary host and prevent them from reaching the secondary host. Regular hand washing is, thus, an excellent way of preventing the transmission of microbes from one person to another and has been described as a modest measure with big effects. Hand washing is especially important where people congregate (schools, offices), where ill or vulnerable people are concentrated (hospitals, nursing homes), where food is prepared and shared and in homes, especially where there are young children and vulnerable adults. ${ }^{2}$

\section{HAND HYGIENE POLICY FOR A TERTIARY CARE HOSPITAL}

Hand hygiene is recognized as the leading measure to prevent cross-transmission of microorganisms. Regarding hospital acquired infections, the compliance of nurses with hand washing guidelines seems to be vital in preventing the disease transmission among patients. There is a paucity of studies exploring this subject in Asia. Especially medical and nursing student's knowledge of 
standard hand hygiene precautions is rarely compared. In one of the study in 2014 by Nair et al revealed that only $9 \%$ of participants (13 out of 144) had good knowledge regarding hand hygiene. Nursing students knowledge $(\mathrm{p}=0.023)$, attitude $(\mathrm{p}=0.023)$, and practices $(\mathrm{p}<0.05)$ were significantly better than medical students.

\section{NEED OF THE STUDY}

Hand hygiene is recognized as the leading measure to prevent cross-transmission of microorganisms and to reduce the incidence of healthcare associated infections. Despite the relative simplicity of this procedure, compliance with hand hygiene among healthcare providers is as low as $40 \%{ }^{4}$ To address this problem, continuous efforts are being made to identify effective and sustainable strategies. Nurses constitute the largest percentage of the healthcare workers (HCWs) and they are the 'nucleus of the healthcare system'. The importance of hands in the transmission of hospital because they spend more time with patients than any other HCWs, their compliance with hand washing guidelines seems to be more vital in preventing the disease transmission among patients. Infections has been well-demonstrated, and can be minimized with appropriate hand hygiene. However, compliance with hand washing is frequently suboptimal. In the Sri Lankan theater settings a study demonstrated that only $60 \%$ of the doctors performed appropriate hand washing before entering the theater. ${ }^{3}$ Noncompliance with hand washing may be due to a variety of reasons, including lack of appropriate facilities for hand washing, high staff to patient ratios, insufficient knowledge and attitudes of the staff, and allergies to hand washing products. Therefore, it is important to address these issues.

\section{METHODOLOGY}

It was a descriptive cross-sectional study carried out between April and August 2013. The study population included Doctors, Nursing personnel, Paramedical staff and quality managers of tertiary care hospital from public and private hospitals. Checklist was made after an exhaustive review of literature which was then improvised. Validation of the checklist was done by experts in infection control in various private and public hospitals. Subsequently, interaction was done with study population against the back drop of the checklist and hand hygiene policy was formulated.

\section{ANALYSIS AND RESULTS}

One hundred people which included doctors, nurses, paramedical staff, pharmacists and quality managers of tertiary care public and private hospitals were approached for interaction against the back drop of the checklist. Total response rate is $56 \%$. Twelve doctors responded out of 20 doctors approached, 18 nurses responded out of 20, 12 quality managers interacted out of 20 and 4 doctors, expertize in infection control practices responded out of 20 approached and 10 nurses, expertize in infection control practices out of 20 approached. Policy was framed after incorporating inputs from responses received against the back drop of the checklist.

\section{HAND HYGIENE POLICY FOR A TERTIARY CARE HOSPITAL}

\section{Aim of the Policy}

To make clear to all the staff about hand hygiene and to make available to the point information and guidance regarding hand hygiene. This document summarizes the information to patients and the public about hand hygiene. It sets out how the institute will meet its training prerequisites to ensure that staff receives adequate training in relation to hand hygiene, thus, reducing the risk of healthcare-associated infection.

\section{GOALS AND PURPOSE}

The goals and purpose of this policy is to ensure that staff pursues proper evidence-based hand washing techniques for the prevention and control of infections. This policy has been developed to lessen the risk of disease transmission to patients via the hands of staff.

\section{SCOPE}

It includes all procedures that require hand hygiene to be maintained.

\section{INTRODUCTION}

Hand hygiene: ${ }^{4}$ Performing hand washing, antiseptic hand wash, alcohol-based hand rub, surgical hand hygiene/ antisepsis.

Hand washing: Washing hands with plain soap and water. Antiseptic hand wash: Washing hands with water and soap or other detergents containing an antiseptic agent.

Alcohol-based hand rub: ${ }^{4}$ Rubbing hands with an alcoholcontaining preparation.

Surgical hand hygiene/antisepsis: Hand washing or using an alcohol-based hand rub before operations by surgical personnel.

Improper hand hygiene by HCWs is responsible for about $40 \%$ of nosocomial infections. Lack of knowledge and lack of recognition of hand hygiene opportunities during patient care are mainly responsible for poor 
hand hygiene among HCWs. Although many countries have guidelines regarding hand hygiene for healthcare settings, overall compliance among HCWs remains poor despite hand hygiene being regarded as one of the most important elements of infection control activities. World Health Organization (WHO), in 2005 issued guidelines regarding specific steps and procedures to be followed during hand washing. ${ }^{5}$ The spread of infections in developing countries remains a serious problem, especially in high-risk settings, such as healthcare facilities due to lack of awareness in HCWs and compounded by 'omo syndrome' (a belief that they are super clean and sterile). ${ }^{8}$ Present study attempts to formulate policy for tertiary care hospitals.

\section{RESPONSIBILITIES}

Responsibility of a hospital/institute are:

- To promote compliance with best practice in hand hygiene

- To embed hand hygiene as an integral part of the organization culture as a matter of clinical governance

- To view any drift in hand hygiene practices as a serious clinical issue

- To ensure all new employees receive the hand hygiene policy

- To make available sufficient resources to facilitate compliance with the hand hygiene policy

- To actively encourage compliance with the hand hygiene policy

- To ensure that audits of hand hygiene compliance are undertaken according to the infection prevention and control team audit calendar within their sphere of management

- To ensure that the amenities and equipment for hand hygiene are in place so that staff have appropriate and convenient access.

Responsibility of Infection Control Committee and Infection Control Team are:

- To counsel on current best practice in hand hygiene policy

- Advise on best practice in planning hand hygiene facilities for new construction and refurbishment work

- Plan and deliver a program of hand hygiene education

- Monitor compliance with the hand hygiene policy through infection control audit and routine observation of practice.

To use routine observation of hand hygiene practices as clinical indicator and feedback via the infection prevention and control link team and the infection control committee.

\section{Responsibility of Infection Control Nurse}

Act as a role model promoting good hygiene practice within the institute. Monitors the progress of hand hygiene assessments undertaken within their clinical areas and put forward support as required.

\section{Responsibility of Nurses' In-Charge}

Make sure that all facilities and equipment are in place to facilitate hand washing.

Any concerns to be highlighted to the member of the infection prevention and control team.

Ensures that all staff within their designated area of responsibility is aware of the hand hygiene policy and have had appropriate hand hygiene training.

Nominate a designated member of the ward staff who ensures that alcohol hand rubs are in place and that dispensers are full and functional.

Make sure that information and posters are available and placed in positions of prominence for all visitors to the ward area to see.

Monitor hand hygiene is taken seriously by all members of the healthcare team and any breaches are dealt with effectively and if necessary as a clinical incident.

Ensure that staff actively participate in all aspects of the Clean Your Hands Campaign.

\section{Individual Responsibility}

Timely effective hand hygiene is the personal responsibility of all individuals involved in the provision of healthcare.

All HCWs have a personal responsibility to comply with all aspects of the hand hygiene policy.

\section{RECOMMENDATIONS}

\section{Indications for Hand Washing and Hand Antisepsis}

- When hands are visibly dirty or contaminated with proteinaceous material or are visibly soiled with body fluids, wash hands with either a non-antiseptic soap and water, or an antiseptic soap and water. ${ }^{6}$

- If hands are not visibly soiled, use an alcohol-based hand rub for routinely decontaminating hands in following situations.

- Before having direct contact with patients.

- Before inserting indwelling urinary catheters, peripheral vascular catheters, or other invasive devices that do not require a surgical procedure.

- After contact with a patient's intact skin (e.g. when taking a pulse or blood pressure, and lifting a patient). 
- After contact with body fluids or excretions, mucous membranes, nonintact skin, and wound dressings if hands are not visibly soiled.

- After removing gloves.

- Wash hands with nonantimicrobial soap and water or with antimicrobial soap and water if exposure to Bacillus anthracis is suspected or proven. The physical action of washing and rinsing hands under such circumstances is recommended because alcohols, chlorhexidine, iodophors, and other antiseptic agents have poor activity against spores.

- After using the toilet

- After cleaning up any spillage

- Before handling food

- Before and after aseptic procedures

- After handling laundry and waste

- Before and after administering medication

- Before and after emptying urinary drainage bags

\section{Types of Hand Hygiene ${ }^{6}$}

\section{Routine Hand Wash}

Agent: Used is soap and water

Purpose: Remove soil, remove transient flora

Indication: Before significant contact with a patient like before emptying a catheter bag, injection, venipuncture, changing a nappy, assisting to eat, between performing procedures on the same patient, after activities likely to cause significant contamination, e.g. direct contact with body secretions, mucous membranes, wounds, removing gloves.

\section{Technique}

- Remove hand and wrist jewellery

- Wet hands first with water

- Apply product as recommended to hands

- Rub hands together vigorously for at least 15 seconds

- Cover all surfaces of the hands and fingers

- Rinse hands with water

- Dry thoroughly with a towel

- Use towel to turn off the faucetor elbow taps if available.

\section{Antiseptic Hand Rubs}

Agent: Used alcohol-based hand rub (alcohol $70 \%$ or alcohol $70 \%$ and triclosan).

Purpose: To destroy transient flora, reduce resident flora.

Note: This type of hand washing will not remove or denature soil.

\section{Indication}

- As for routine hand wash

- May be performed in lieu of routine hand wash, but only if hands are free of visible soil.

- May be performed in emergency situations where there is insufficient time/facilities (water).

\section{Technique}

- Remove hand and wrist jewellery

- Apply product as recommended to hands

- Rub hands together vigorously for at least 15 seconds

- Thoroughly rub all surfaces of the hands and fingers until hands are dry.

\section{Antiseptic Hand Wash}

Agent: Antiseptic soap, water friction.

Purpose: Remove soil, remove transient rlora, reduce resident flora.

Indication: Routine hand wash may be the preferred type of hand decontamination when attending high risk patient, e.g. neonatal intensive care unit (NICU), pediatric intensive-care unit (PICU), oncology, central lines but this type of hand hygiene can also be used.

\section{Technique}

- Remove hand and wrist jewellery

- Wet hands first with water

- Apply product as recommended to hands

- Rub hands together vigorously for at least 15 seconds

- Cover all surfaces of the hands and fingers

- Rinse hands with water

- Dry thoroughly with a towel.

Note: Hand antisepsis occurs simultaneously with hand washing when soaps for detergents which contain antiseptics are used.

\section{Clinical Hand Wash}

Agent: Used antiseptic soap, water friction

Purpose: To remove soil, remove transient flora, reduce resident flora.

Indication: Nonsurgical procedures which require aseptic technique, e.g. peripheral venous cannulation, insertion of a urinary catheter, wound dressings.

\section{Technique}

- Remove hand and wrist jewellery

- Wet hands first with water

- Apply product as recommended to hands

- Rub hands together vigorously for at least 60 seconds

- Cover all surfaces of the hands and fingers 
- Rinse hands with water

- Dry thoroughly with a towel.

\section{Surgical Hand Antisepsis}

Agents used: Antiseptic soap only or soap and then alcohol based hand rub with persistent activity.

Purpose: To remove soil, transient flora reduce resident flora for duration of surgery in care of glove tear.

Indication: All surgical procedures including insertion of all CVC lines.

\section{Technique}

- Remove rings, watches, and bracelets before beginning the surgical hand scrub

- Fingernails should be kept short and well maintained

- Hands and forearms must be free of open lesions and breaks in skin integrity

- Be wearing complete operating room attire including mask, cap, and goggles if they are to be worn

- Keep clothing away from sink and splashes

- Turn on water and adjust temperature for your comfort

- Keep arms level well away from body and hands up above elbows for duration of scrub

- Wet hands and forearms

- Apply antiseptic hand wash solutions

- Lather hands and forearms for at least one minute from fingertips to three inches above elbows starting with hands to forearm, forearm to elbow

- Wash hands thoroughly, using the following steps to facilitate eradication of all bacteria and 10 seconds/ step. are:

Seven-step hand washing technique is being followed

1. Step 1: Palm to palm

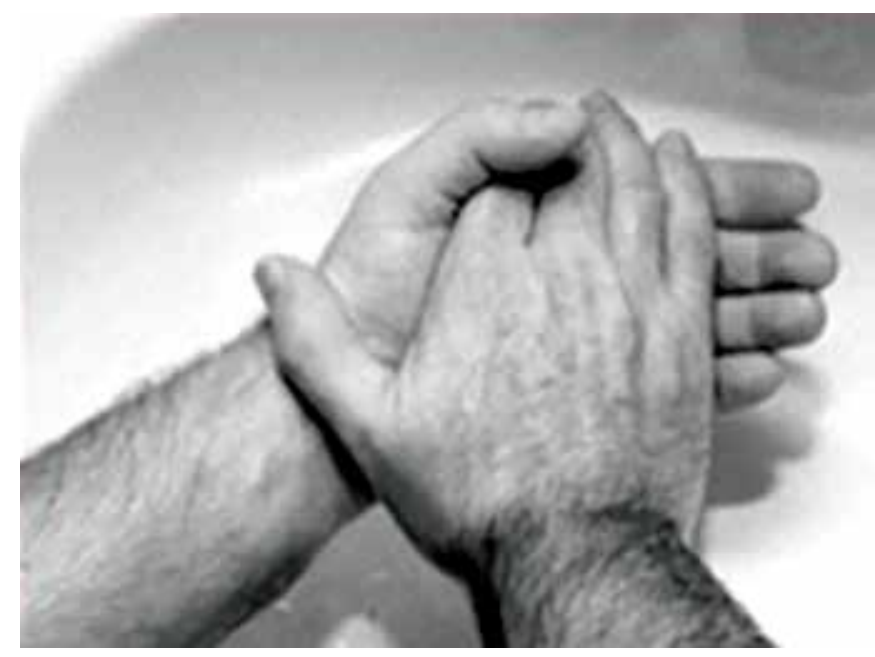

2. Step 2: Right palm over back of left hand. Change hands and repeat.

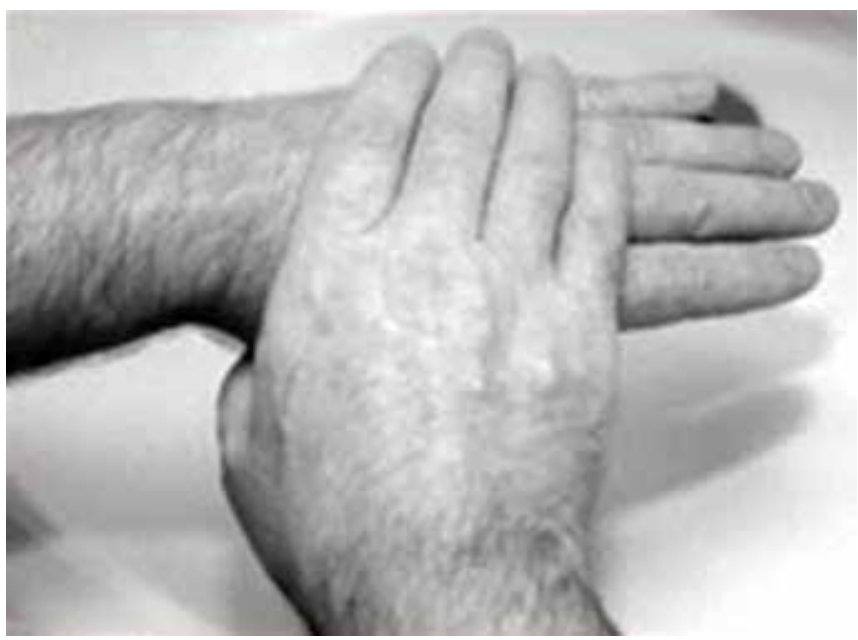

3. Step 3: Interlace fingers of right hand over left. Change hands and repeat.

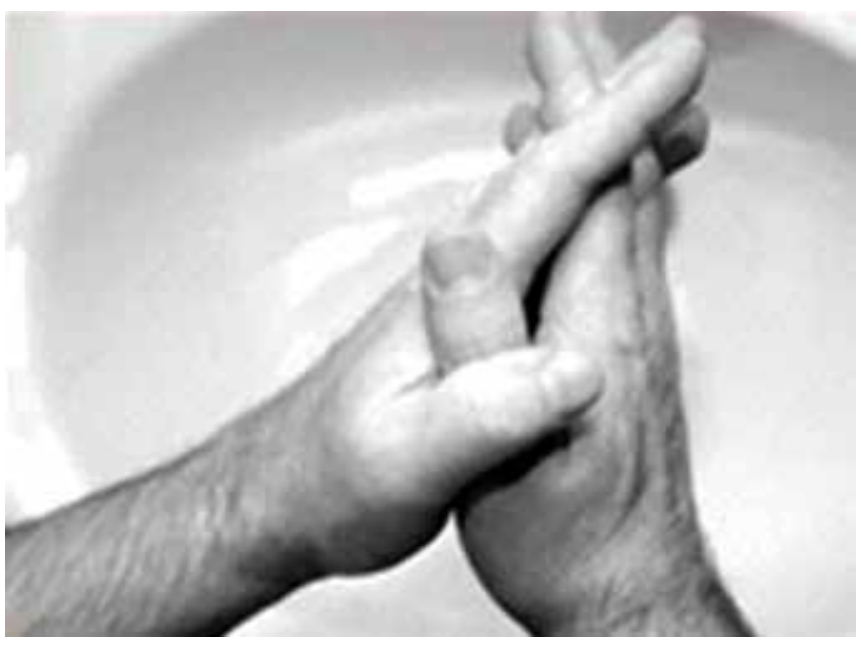

4. Step 4: Rotational rubbing backward and forward with clasped fingers of right hand in left palm. Change hands and repeat.

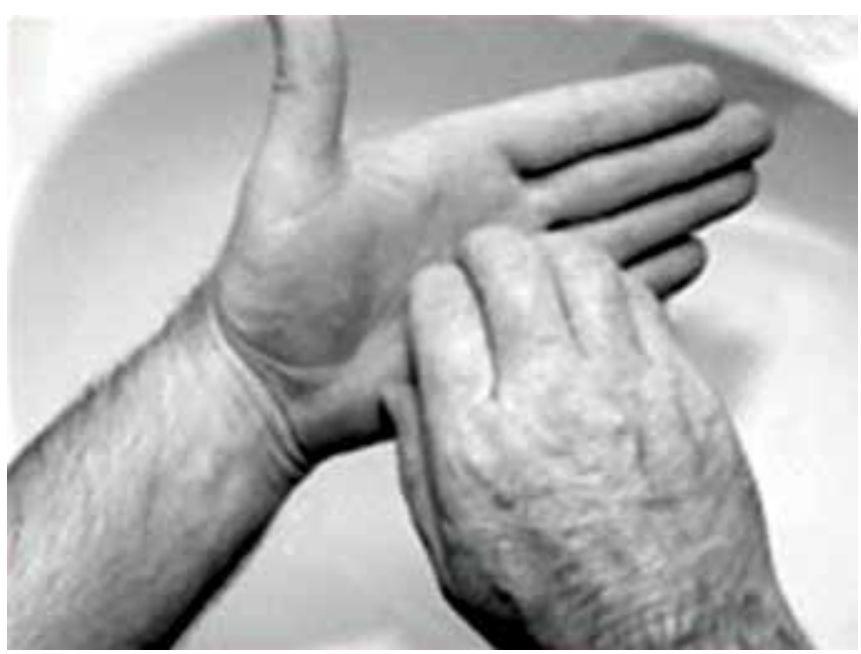


5. Step 5: Rotational rubbing of right thumb clasped in left palm. Change hands and repeat.

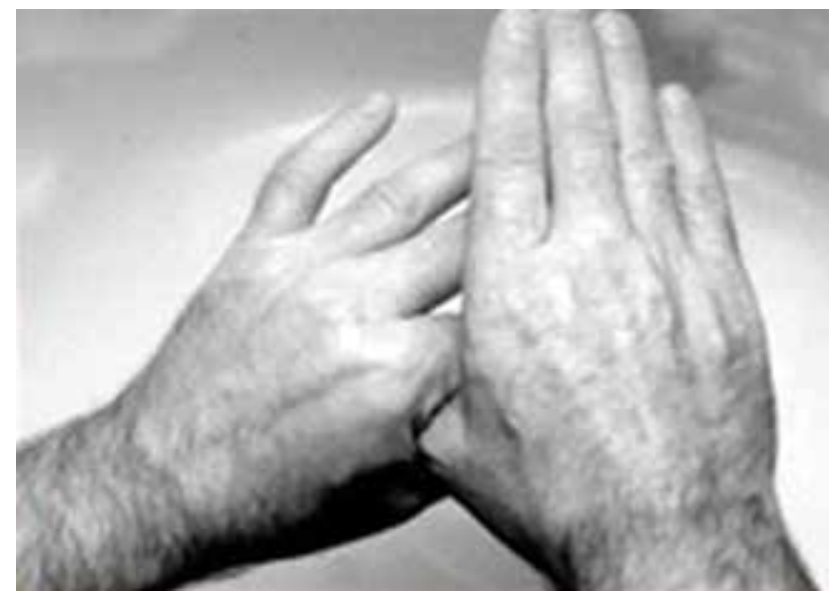

6. Step 6: Grasp left wrist with right hand and work cleanser into skin. Change hands and repeat.

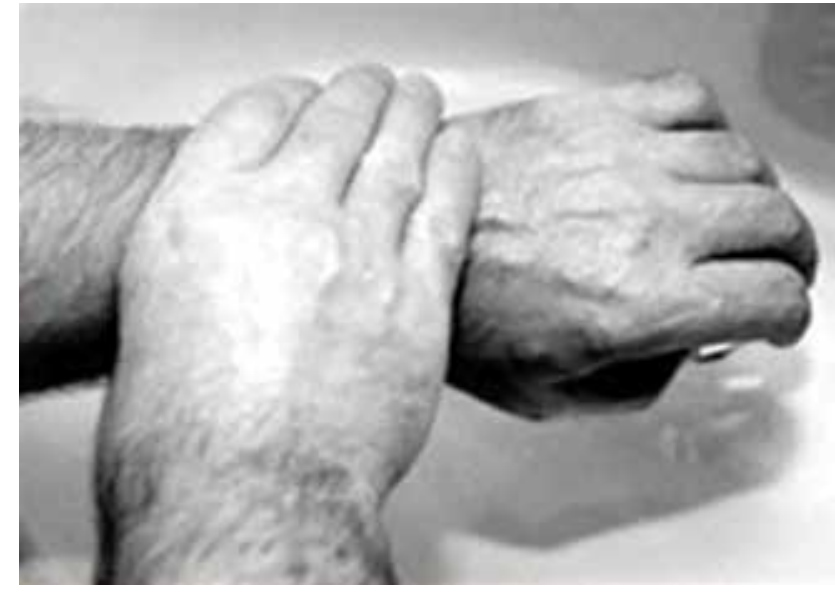

7. Step 7: Rub hands and wrists for 30 seconds, then rinse and dry thoroughly.

- Apply antiseptic hand wash solution a second time

- Lather hands and forearms for at least 2 minutes in the same manner

- Recommended scrub time is between 2 and 6 minutes, longer times are not necessary

- Prolonged and abrasive scrubbing may damage the skin

- Pay attention to all areas of the hands and forearms

- Rinse hands and forearms under running water keep hands higher than the elbow at all times

- Thoroughly dry hands and forearms with a sterile towel keeping hands raised.

Proceed to OT keeping hands above the elbow and out from scrub clothes.

- Allow hands and forearms to dry thoroughly before donning sterile gloves.

- Between short cases only, hands may be disinfected by using two or more applications of an alcohol hand rub.
Before applying the alcohol solution, pre wash hands and forearms with a nonantiseptic soap and dry hands and forearms completely.

\section{Selection of Hand Hygiene Agent ${ }^{4}$}

- To provide with hand-hygiene products that have low irritancy potential, mainly when these products are used numerous times per shift.

- When selecting nonantimicrobial soaps, antimicrobial soaps, or alcohol-based hand rubs, solicit information from manufacturers regarding any known interactions between products used to clean hands, skin care products, and the types of gloves used in the institution.

- Before making purchasing decisions, evaluate the dispenser systems of various product manufacturers or distributors to ensure that dispensers function adequately and deliver an appropriate volume of product.

- Central infection control council recommends that either Micro shield or Sterillium can be used as an alcohol-based hand rub. In OTs, only micro shield should be used because of greater residual activity.

- Do not add soap to a partially empty soap dispenser. This practice of 'topping off' dispensers can lead to bacterial contamination of soap'.

\section{Other Important Aspects of Hand Hygiene ${ }^{4}$}

Avoid wearing artificial fingernails or extenders when in direct contact with patients at high risk (e.g. those in intensive-care units or operating rooms).

- Keep natural nails tips less than 1/4" long

- To wear gloves when contact with blood or other potentially infectious materials, mucous membranes, and non intact skin could occur.

- To remove gloves after caring for a patient. Do not wear the same pair of gloves for the care of more than one patient, and do not wash gloves between uses with different patients.

- To change gloves during patient care if moving from a contaminated body site to a clean body site.

\section{Facilities}

All clinical areas viz. consultation chambers, nursing stations and critical care areas should have:

- Hand washing facilities appropriate to the area

- Clear unobstructed access to the hand washing sink

- Hand washing sinks for that purpose only and clear of inappropriate items

- Liquid soap and alcohol hand rubs available at every sink 
- Hand drying facilities with disposable paper towels must be readily available at every sink

- Hand washing posters should be placed by each sink

- All critical areas should have alcohol-based hand rubs installed at entry points for use by visitors

- All critical areas should have alcohol-based hand rubs by each patient bedside

- The infection control committee should be consulted before any new construction or refurbishment work is planned to advice on sink type, number and placement of hand washing facilities.

\section{Hand Washing Agents ${ }^{6}$}

There are three types of agent, which can be used to remove microorganisms from hands: soap, alcohol-based, hand rubs and antimicrobial agents.

\section{Soap}

Will mechanically remove transient microorganisms but has little effect on resident microorganisms.

\section{Alcohol-based Hand Rubs}

Can be applied quickly without access to water. However, they are not effective in removing soiling and should only be used if hands are visibly clean.

Recent studies advocate the use of alcoholic hand rubs between each patient contact as a measure, which reduces the rate of hospital-acquired infection.

\section{Antiseptic Agents}

Are designed to remove transient and reduce resident skin microorganisms. Chlorhexidine-based preparations have been found to be more effective than iodine-based solutions as they have a residual effect, which influences the survival times of many organisms on hand surfaces.

Antimicrobial agents should be used in situations when there is a need to reduce resident microbial flora, e.g. in operating theaters or similar departments or when dealing with patients in isolation and before performing an invasive procedure.

\section{Hand Drying Agents}

Drying hands with paper products is preferable to using hot air or linen towels. The use of hot air dryers should not be used in clinical areas as these spread airborne bacteria by recirculating the surrounding environmental air.

Drying with a high absorbency paper towel will remove some of the transient organisms that remain after hand washing. Paper towels should be wall mounted.

\section{Patient Hand Hygiene}

Hand hygiene for patients must be encouraged as it is equally as important in the prevention and control of infection. Staff must ensure that patients are afforded an opportunity to hand wash prior to meals, after having used a bedpan/urinal or toilet or when hands are otherwise soiled.

\section{Quality Assurance}

- Training to be made mandatory for newly joined staff on hand hygiene

- Continuous monitoring and record keeping of adherence to hand hygiene practices

- Mechanism to provide feedback to healthcare workers about their performance

- To monitor the quantity of alcohol-based hand rub used per 1,000 patient days

- To monitor hospital acquired infection rates.

\section{REFERENCES}

1. Ekwere TA, Okafor IP. Hand hygiene knowledge and practices among healthcare providers in a tertiary hospital, South West Nigeria. Int J Infect Control 2013;9(4):1-10.

2. Harcourt P. Hand washing practices amongst medical students in Port Harcourt, Nigeria. Niger Heal J 2009;9(1): 16-20.

3. Nair SS, Hanumantappa R, Gurushantswamy S, Siraj MA, Raghunath P. Knowledge, Attitude, and Practice of Hand Hygiene among Medical and Nursing Students at a Tertiary Healthcare Centre in Raichur, India. ISRN Prev Med 2014;2014:1-4.

4. Boyce JM. Pittet CD. Guideline for hand hygiene in health care settings. Infect Dis Clin Pract 2002;11(5):306-311.

5. Anargh LV, Harpreet M, Kulkarni A, Kotwal CA. Hand hygiene practices among healthcare workers (HCWs) in a tertiary care facility in Pune 2012;9(0):8-10.

6. Larson E. APIC Guideline for hand washing and hand antisepsis in health-care settings*. Am J Infect Control 1995;23(4):251-269.

7. Doebbeling BN, Stanley GL, Sheetz CT, et al. Comparative efficacy of alternative handwashing agents in reducing nosocomial infections in intensive care units. N Engl J Med 1992;327:88-93.

8. Rotter ML, Koller W. Surgical hand disinfection: effect of sequential use of two chlorhexidine preparations. J Hosp Infect 1990;16:161-166.

9. Ehrenkranz NJ. Bland soap handwash or hand antisepsis? The pressing need for clarity. Infect Control Hosp Epidemiol 1992;13:299-301.

10. Ehrenkranz NJ, Alfonso BC. Failure of bland soap handwash to prevent hand transfer of patient bacteria to urethral catheters. Infect Control Hosp Epidemiol 1991;12:654-662.

11. Larson E. APIC guideline for use of topical antimicrobial agents. Am J Infect Control 1988;16:253-266.

12. Association of operating room nurses. Recommended practices: surgical hand scrubs. Aorn J 1990;52:830-836. 